\title{
Thermochromatism and Structural Evolution of Metastable Polydiacetylenic Crystals
}

Jiebin Pang, Lu Yang, Byron F. McCaughey, Huisheng Peng, Henry S. Ashbaugh, C. Jeffrey Brinker, and Yunfeng Lu
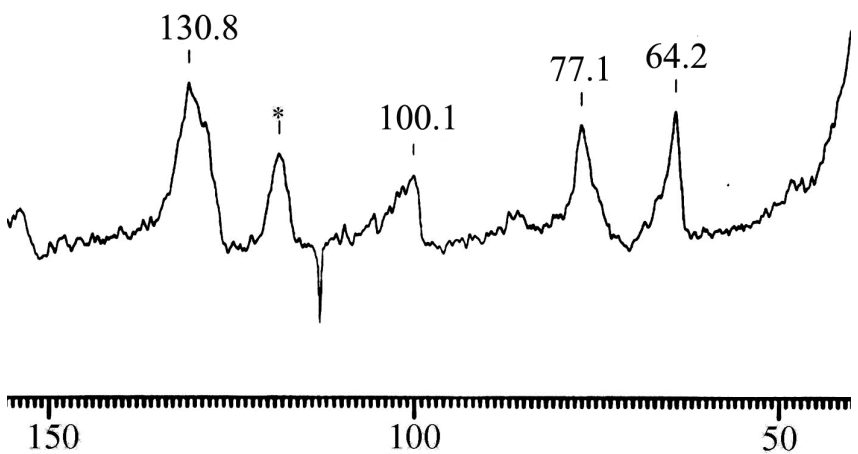

Figure SI-1. Partial ${ }^{13} \mathrm{C}$ CP/MAS NMR spectrum of the PDA-Na microcrystals polymerized by UV radiation. Asterisk indicates the spinning sideband. The presence of peak at $130.8 \mathrm{ppm}$ corresponding to the $\mathrm{sp}^{2}$ olefinic carbons of the PDA backbones suggests polymerization of the diacetylene units. The chemical shift at about $100.1 \mathrm{ppm}$ can be assigned to the acetylenic carbons of the PDA backbones. The chemical shifts at 77.1 and $64.2 \mathrm{ppm}$ assigned to the outer and interior acetylenic carbons in the residual unreacted monomers indicate a partial polymerization of PCDA. Estimating from the peak heights of the reacted and unreacted acetylenic carbons, the degree of polymerization is higher than $50 \%$.
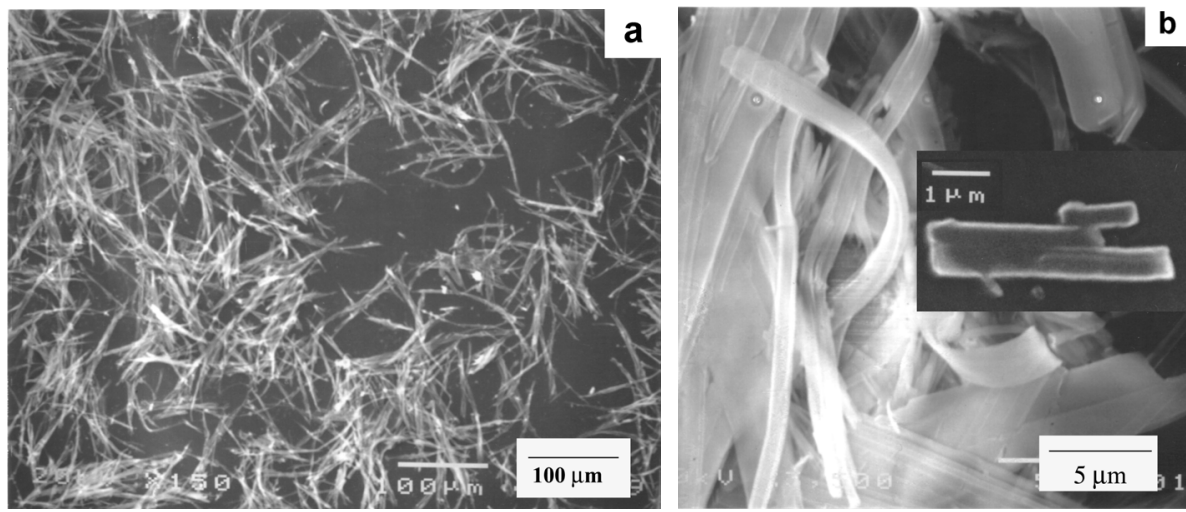

Figure SI-2. Representative SEM micrographs of the blue PDA-Na microcrystals showing a long, strip-like morphology. The inset in (b) shows an image of several small single crystals. 
Supporting Information for J. Phys. Chem. B

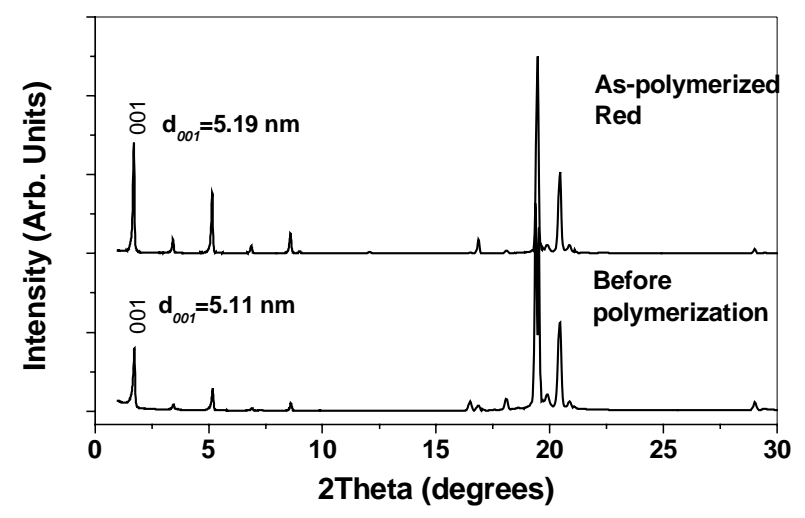

Figure SI-3. XRD patterns for the PCDA-Na microcrystals before and after topochemical polymerization. They show similar crystalline structures. Note that, however, some polymerization occurred under X-ray radiation during the scanning. This strongly supports that the molecular packing structure does not change significantly during the polymerization at room temperature. 